\title{
Proteomic and metabolomic advances uncover biomarkers of mitochondrial disease pathophysiology and severity
}

\author{
Marjan Gucek' and Michael N. Sack ${ }^{2}$ \\ 'Proteomics Core and 'Laboratory of Mitochondrial Biology and Metabolism National Heart, Lung and Blood Institute, NIH, Bethesda, Maryland, USA.
}

\begin{abstract}
Advancing proteomic and metabolomic technologies that integrate curated omic databases have crossed a threshold to enable their clinical utility. In this issue of the $J C l$, Sharma et al. exploit emerging technologies to evaluate whether biomarkers identified in the mitochondrial encephalomyopathy lactic acidosis and stroke-like episodes (MELAS) syndrome could refine disease characterization, uncover pathways to monitor therapeutic efficacy, and/or delineate disease-modifying targets. The authors analyzed blood and urine samples from patients with this genetic mitochondrial disease and elucidated proteins and metabolites related to NADH-reductive stress. These circulating biomarkers have intriguing clinical potential that implicate disease pathophysiology and may prove important biomarkers for the future management of MELAS.
\end{abstract}

\section{Evaluating mitochondrial function}

Genetic mitochondrial diseases arise from mutations in nuclear genes that encode mitochondrial functioning proteins or directly from point mutations or deletions in the mitochondrial genome. Mitochondrial DNA (mtDNA) mutations add complexity to disease diagnosis, presentation, and progression since the proportion of healthy to mutated mitochondrial nucleoids within each cell can vary (heteroplasmy), a characteristic that can extend to the tissue level and vary by individual. The effect of heteroplasmy and tissue involvement is exemplified in Pearson marrow-pancreas syndrome where mtDNA deletion results in a disease spectrum that includes congenital anemia with bone marrow failure, infectious susceptibility, and varying degrees of involvement of the pancreas, liver, endocrine system, kidneys, and central nervous system (1).
The dynamic role of heteroplasmy in this syndrome is evidenced by the improvement of transfusion-dependent anemia over time. The exact mechanisms whereby the proportion of postembryogenesis heteroplasmy is reduced has not been clearly delineated, but is more apparent in rapidly dividing cells where a preferential quality control program may orchestrate removal of mutated mtDNA (2). Similarly, the loss of mtDNA heteroplasmy is also observed in the blood of aging patients that have mitochondrial encephalopathy lactic acidosis and stroke-like episodes (MELAS) syndrome (3).

Although next-generation DNA sequencing has markedly improved the ability to diagnose mitochondrial genetic disorders (4), the role of heteroplasmy and the different tissue segregation of mtDNA perturbations may restrict the role of genomics in predicting disease spectrum, severity, or progression. The question

Related Article: https://doi.org/10.1172/JCl136055

Conflict of interest: The authors have declared that no conflict of interest exists.

Copyright: () 2021, American Society for Clinical Investigation.

Reference information: J Clin Invest. 2021;131(2):e145158. https://doi.org/10.1172/JCI145158.

arises as to whether the determination of mitochondrial function may add insight into these diseases with potential clinical utility. As mitochondria play central roles in energetics, iron and cholesterol metabolism, and redox handling, the exploitation of advancing mass spectrometry and newer omic technologies could uncover distinct serological biochemical profiles that reflect aspects of mitochondrial perturbations that may be linked to distinct mtDNA pathology.

The technical challenge of depleting serum from dominating abundant proteins, which occur in the range of 10 orders of magnitude, to identify less abundant proteins, has historically limited proteomic studies and required using liquid chromatography coupled with mass spectrometry (LC-MS). Recent technologies have emerged, including SOMAscan (an aptamer-based assay using short oligonucleotides with single-protein binding affinity) or the Olink protein platform (oligonucleotide-labeled antibodies) to measure in excess of 1000 proteins in serum without the need for depletion $(5,6)$. Both of the affinity-based platforms enable unparalleled throughput in the clinical setting, however, they have drawbacks in that they only quantify predetermined targets, may have diminished affinity for posttranslational modifications or protein degradation products, and may have low affinity for mutated protein products or polymorphisms.

Nuclear magnetic resonance spectroscopy, gas chromatography-mass spectrometry (GC-MS), and LC-MS spectral libraries for targeted metabolomics continue to expand. Concurrently, the Human Metabolome Database is frequently curated to add spectral and chemical properties of newly defined metabolites, thereby enabling investigators to use untargeted approaches to identify metabolite alterations under different pathophysiologic 


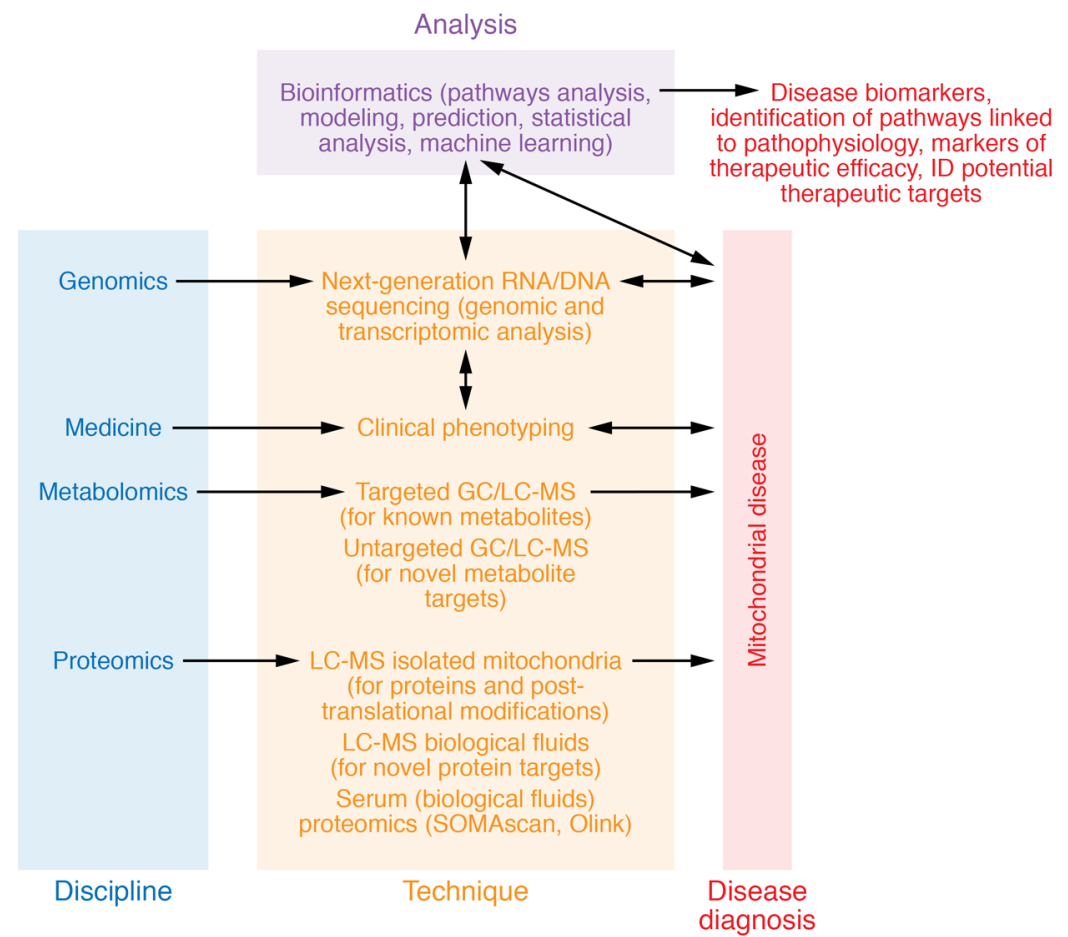

Figure 1. The integration of omic approaches with clinical phenotyping, bioinformatics tools, and databases. Disease discovery and therapeutic opportunities are advanced by next-generation transcript and genome sequencing and a wide array of bioinformatics approaches. LC-MS can identify previously unknown proteins and proteins with posttranslational modifications from biological fluids or isolated mitochondria. Similarly, GC-MS can identify known or unique metabolites. The Olink protein platform is a technology that uses oligonucleotide-labeled antibodies and can measure over 1000 proteins in serum without the need for depletion. Many diseases are categorized based on phenotypic features then diagnosed using next-generation RNA or DNA sequencing. In parallel, proteomic and metabolomic analysis can identify novel targets linked with the disease. Subsequent bioinformatics analysis can curate and integrate these targets to uncover pathophysiologic pathways linked to disease and to identify putative therapeutic approaches. Sharma et al. measured more than 1000 analytes from the serum of patients with MELAS using SOMAscan, an aptamer-based assay that uses short oligonucleotides with single-protein binding affinity and LC-MS (8). The researchers identified more than 20 metabolites that also coincided with NADH redox imbalance.

conditions (7). Together, these untargeted approaches cover a wide range of metabolite polarity to identify the diverse metabolite pool that is present in serum (Figure 1).

\section{Employing emerging technologies}

In this issue of the JCI, Sharma et al. employ emerging technologies to identify proteins and the metabolic signature of one of the most frequent inherited mitochondrial diseases, MELAS syndrome, which harbors a heteroplasmic m.3243A $>$ G mutation in the MT-TL1 gene encoding mitochondrial leucyl-tRNA 1 (8). As with other mtDNA-linked diseases, MELAS has a spectrum of presentations, and patients with these mutations have a higher prevalence of other comorbidities. As would be expected, the disease pro- gression is unpredictable, although once stroke-like episodes develop, the progression is relentless, with subsequent diminished life expectancy (9). The actual incidence of the m.3243A $>G$ variant is high at around 1 in 400 individuals, although the prevalence of MELAS itself approximates 0.2 per 100,000 adults $(10,11)$. To date, investigations include magnetic resonance spectroscopy (MRS) to measure brain lactate levels, biochemical analysis of mitochondrial function in tissue biopsies, and serum lactate, urine heteroplasmy and quantification of multisystemic deficits using the Karnofsky performance status score. In an attempt to refine our ability to characterize the disease, Sharma et al. employed targeted and untargeted plasma proteomic and metabolomic biomarker approaches in two phases: the discovery cohort, which included control subjects, m.3243A > G variant/obligate carrier individuals, with a range of disease features excluding stroke-like episodes, and m.3243A > G MELAS patients with established stroke-like episodes; and the validation cohort, which included the same categories. The researchers compared the findings to the current diagnostic array of studies (8).

Plasma proteomics was explored using the SOMAscan platform and, once controlled for known age, sex, and BMI variables, four proteins met the FDR threshold that were distinct between controls and MELAS patients (Figure 1). The most highly differentiated protein was the previously identified growth differentiation factor 15 (GDF-15). The other three proteins matched profiles in the bioinformatic database and were considered previously unidentified markers of disease. Interestingly, one of these proteins, the receptor tyrosine kinase (RET), displayed the highest positive correlation with the Karnofsky score. Other differentiated proteins failed to uncover putative mitochondrial diseaselinked biological pathways (8).

When compared with other proteomic analysis, LC-MS more successfully identified differences between polar and nonpolar metabolite groups after controlling for the same variables and employing the same FDR threshold. The targeted metabolomic analysis validated prior findings of changes in multiple metabolites linked to glycolysis, the tricarboxylic acid cycle, fatty acid oxidation, and the electron transfer chain. Of greater interest, untargeted metabolomics harnessed changes in spectral peaks, with the comparisons with exact masses in the Human Metabolome Database, to identify three metabolite families as candidates that distinguished patients with MELAS from controls. The biological role of the N-lactoyl-amino acid family of intermediates remains unclear. In contrast, the elevation of $\beta$-hydroxy acylcarnitines (BOHCAs) and the $\beta$ hydroxy fatty acids (BOHFAs) analytes support parallel perturbations in hydroxylated acylcarnitines and fatty acids, which may share a common biochemical origin. Furthermore, Sharma et al. postulated that this hydroxylation in the $\beta$ position may disrupt $\mathrm{NAD}^{+}$-dehydrogenation enzymes, resulting in an increased $\mathrm{NADH} / \mathrm{NAD}^{+}$ 
ratio depicting $\mathrm{NADH}$ redox imbalance or NADH-reductive stress. This NADHimbalance hypothesis is supported in part by the targeted metabolomic data showing accumulation of intermediates upstream of $\mathrm{NAD}^{+}$-dependent enzymes in the glycolytic, fatty acid $\beta$-oxidation and TCA cycles and in ketone metabolism with the elevation of $\beta$-hydroxybutyrate upstream of its dehydrogenase (8).

Sharma and authors then compared the overall proteomic and metabolomic data sets from the 3 groups with the canonical measures of disease severity and validated that the putative markers and metabolic analytes correlated very well with all the standard investigational studies. As the Karnofsky score is the best composite of disease severity, it was notable that RET and BOHFAs and BOHCAs were highly correlated with this score, and outperformed other standard investigational assays (8). At the same time, the analysis of plasma from a prior study in controls and patients with MELAS at baseline and in response to exercise confirmed that $\alpha$ hydroxybutyrate, N-lactoyl-amino acids, and distinct hydroxy-fatty acids were elevated in patients with MELAS and were less labile compared with lactate in response to exercise (12). A stableisotope dilution method was then employed with targeted and untargeted metabolites. Notably, quantitative metabolite levels could distinguish between the MELAS and 3243A $>G$ variant patients and thereby reflect disease severity (8).

\section{Conclusions and considerations}

Although it is unlikely that omics-based analytics will replace MRS brain lactate measurements and the Karnofsky functional score in assigning disease severity, metabolites revealed by Sharma et al. may serve as biomarkers that assess therapeutic efficacy or identify therapeutic pathways (8). For example, metabolite markers may reflect therapeutic responses to strategies that diminish $\mathrm{NAD}^{+}$-reductive stress (13, 14) and/or augment mitochondrial fidelity and function $(15,16)$ for MELAS and other genetic mitochondrial pathologies.
At the same time GDF-15 and RET, both identified as distinguishing biomarkers of MELAS, are in the same anorexic-response signaling pathway and the loss-of-function RET mutation is linked to bowel obstruction in Hirschsrung disease (17). Hence, clinicians may consider targeting GDF-15 and RET pathways to ameliorate gastrointestinal symptoms, including vomiting and pseudo-obstruction in patients with MELAS. Conversely, comorbidities linked to MELAS and other genetic mitochondrial defects, including an increased incidence of diabetes and heart failure, may impact the specificity of these omic biomarkers $(18,19)$. These diseases in their own right perturb mitochondrial function and elicit proteomic and metabolomic signatures that may confound the effects of primary mitochondrial defects (20). Nevertheless, Sharma et al. is a pioneering and exemplary study that exploits advancing technologies in proteomics and metabolomics to characterize disease, evaluate therapeutic efficacy, and potentially identify therapeutic targets in previously unspecified biological pathways (8).

\section{Acknowledgments}

This work was funded by the Division of Intramural Research of the National Heart, Lung and Blood Institute of the NIH (ZIA-HLO05102 to MNS).

Address correspondence to: Michael N. Sack, National Heart Lung and Blood Institute, NIH, Building 10-CRC, Room 5-3342, 10 Center Drive, Bethesda, Maryland 20892, USA. Phone: 301.402.9259; Email: sackm@nih.gov.

1. Farruggia $P$, et al. Pearson Syndrome: a retrospective cohort study from the marrow failure study group of A.I.E.O.P. (Associazione Italiana Emato-Oncologia Pediatrica). JIMD Rep. 2016;26:37-43.

2. Saneto RP, Sedensky MM. Mitochondrial disease in childhood: mtDNA encoded. Neurotherapeutics. 2013;10(2):199-211.

3. Rahman S, et al. Decrease of $3243 \mathrm{~A} \rightarrow \mathrm{G}$ mtDNA mutation from blood in MELAS syndrome: a longitudinal study. Am J Hum Genet. 2001;68(1):238-240.

4. Schmitt MW, et al. Detection of ultra-rare mutations by next-generation sequencing. Proc Natl
Acad Sci U S A. 2012;109(36):14508-14513.

5. Rohloff JC, et al. Nucleic acid ligands with protein-like side chains: modified aptamers and their use as diagnostic and therapeutic agents. Mol Ther Nucleic Acids. 2014;3:e201.

6. Assarsson E, et al. Homogenous 96-plex PEA immunoassay exhibiting high sensitivity, specificity, and excellent scalability. PLoS One. 2014;9(4):e95192.

7. Wishart DS, et al. HMDB 4.0: the human metabolome database for 2018. Nucleic Acids Res. 2018;46(D1):D608-D617.

8. Sharma R, et al. Circulating markers of NADH-reductive stress correlate with mitochondrial disease severity. JClin Invest. 2020:https:// doi.org/10.1172/JCI136055.

9. Kaufmann P, et al. Natural history of MELAS associated with mitochondrial DNA m.3243A $\rightarrow$ G genotype. Neurology. 2011;77(22):1965-1971.

10. Elliott HR, et al. Pathogenic mitochondrial DNA mutations are common in the general population. Am J Hum Genet. 2008;83(2):254-260.

11. Manwaring N, et al. Population prevalence of the MELAS A3243G mutation. Mitochondrion. 2007;7(3):230-233.

12. Delaney NF, et al. Metabolic profiles of exercise in patients with McArdle disease or mitochondrial myopathy. Proc Natl Acad Sci U S A. 2017;114(31):8402-8407.

13. Fujii T, et al. Efficacy of pyruvate therapy in patients with mitochondrial disease: a semi-quantitative clinical evaluation study. Mol Genet Metab. 2014;112(2):133-138.

14. Patgiri A, et al. An engineered enzyme that targets circulating lactate to alleviate intracellular NADH:NAD ${ }^{+}$imbalance. Nat Biotechnol. 2020;38(3):309-313.

15. Khan NA, et al. Effective treatment of mitochondrial myopathy by nicotinamide riboside, a vitamin B3. EMBO Mol Med. 2014;6(6):721-731.

16. Lee CF, et al. Targeting $\mathrm{NAD}^{+}$metabolism as interventions for mitochondrial disease. Sci Rep. 2019;9(1):3073.

17. Mullican SE, et al. GFRAL is the receptor for GDF15 and the ligand promotes weight loss in mice and nonhuman primates. Nat Med. 2017;23(10):1150-1157.

18. Onishi H, et al. Mitochondrial myopathy, encephalopathy, lactic acidosis and stroke-like episodes (MELAS) and diabetes mellitus: molecular genetic analysis and family study. J Neurol Sci.1993;114(2):205-208.

19. Okajima Y, et al. A follow up study of myocardial involvement in patients with mitochondrial encephalomyopathy, lactic acidosis, and stroke-like episodes (MELAS). Heart. 1998;80(3):292-295.

20. De Souza AI, et al. Proteomic and metabolomic analysis of atrial profibrillatory remodelling in congestive heart failure. J Mol Cell Cardiol. 2010;49(5):851-863. 